\title{
An Experimental Programme optimized with Uncertainty Propagation: PETALE in the CROCUS Reactor
}

\author{
Vincent Lamirand ${ }^{1,2^{*}}$, Axel Laureau ${ }^{1}$, Dimitri Rochman ${ }^{2}$, Gregory Perret $^{2}$, Adrien Gruel $^{3}$, Pierre Leconte ${ }^{4}$, Patrick Blaise ${ }^{3}$, \\ and Andreas Pautz ${ }^{1,5}$ \\ ${ }^{1}$ EPFL, Laboratory for Reactor Physics and Systems behaviour (LRS), 1015 Lausanne, Switzerland \\ ${ }^{2}$ PSI, Laboratory for Reactor Physics and Thermal Hydraulics (LRT), 5232 Villigen, Switzerland \\ ${ }^{3} \mathrm{CEA} / \mathrm{DEN}$, Experimental Physics, Safety \& Instrumentation Division (SPESI), 13108 St-Paul-Lez-Durance, France \\ ${ }^{4}$ CEA/DEN, Reactor Physics and Fuel Cycle Division (SPRC), 13108 St-Paul-Lez-Durance, France \\ ${ }^{5}$ PSI, Nuclear Energy and Safety Research Division (NES), 5232 Villigen, Switzerland
}

\begin{abstract}
The PETALE experimental programme in the CROCUS reactor at EPFL intends to contribute to the validation and improvement of neutron nuclear data in the $\mathrm{MeV}$ energy range for stainless steel, particularly in the prospect of heavy reflector elements of pressurized water reactors. It mainly consists of several transmission experiments: first, through metallic sheets of nuclear-grade stainless steel interleaved with dosimeter foils, and, successively, through its elemental components of interest - iron, nickel, and chromium. The present article describes the study for optimizing the response of the dosimetry experiments to the nuclear data of interest.
\end{abstract}

\section{Introduction}

The present article describes an optimization study for the design of PETALE [1], an oncoming experimental programme in the CROCUS reactor. CROCUS is a teaching and research zero-power reactor operated by the Laboratory for Reactor Physics and Systems Behaviour (LRS) at the Swiss Federal Institute of Technology in Lausanne (EPFL). Within the framework of a collaboration with CEA Cadarache, PETALE intends to contribute to the validation efforts on neutron nuclear data in the $\mathrm{MeV}$ energy range for stainless steel, particularly focusing on heavy reflector elements of pressurized water reactors (PWR). It mainly consists of transmission experiments, where part of the water reflector will be replaced by successive sheets of stainless steel alloy and its elemental components natural iron, nickel, and chromium. The metallic sheets will be set in a watertight box in air, and interleaved with thin dosimeter activation foils. In addition, reflectorreactivity-worth estimates, in-core spectra characterisations, and sheet activation mapping, when possible, will be performed. While the experiments with the alloy will be used as a reference, the successive experiments with its main elements aim at avoiding compensation effects with data assimilation methods. [2]

Because of the opposition between the low neutron flux in the dosimeters in the range of interest, requiring irradiations of maximal power and duration, and the operational constraints of the teaching activities at the CROCUS facility, an optimization of the experimental programme was carried out. Reaction rates of interest were maximised, and therefore the sensitivity to target nuclear data, while the integrated power was minimized. The work was performed by propagating uncertainties using the Total Monte Carlo (TMC) approach with ACE files from the TENDL-2017 library and the Serpent2 Monte Carlo code, in association with an acceleration method, Correlated Sampling (CS). [3-5] In this article, the design of the dosimetry experiments optimized with this methodology is presented, starting from the selection of dosimeter materials, to target uncertainties with regards to nuclear data and operation prospects.

\section{Experimental setup}

\subsection{The CROCUS reactor}

CROCUS is an experimental zero-power reactor, uranium-fuelled and water-moderated, dedicated to education in radiation and reactor physics, and to research [6-17]. A complete description of the core can be found in the International Reactor Physics Experiments Handbook (IRPhE) [18].

CROCUS is licensed for operating at a maximum power of $100 \mathrm{~W}$, i.e. $\sim 2.5 \times 10^{9} \mathrm{~cm}^{-2} . \mathrm{s}^{-1}$ total neutron flux at the core centre. Criticality is controlled either by water level using a spillway (see Fig. 1), or two $\mathrm{B}_{4} \mathrm{C}$ absorber control rods, with an accuracy of $\pm 0.1 \mathrm{~mm}$ (equivalent to approximately $\pm 0.4 \mathrm{pcm})$ and $\pm 0.5 \mathrm{~mm}( \pm 0.2 \mathrm{pcm})$, respectively. This precision and water level's linear trend are of interest for the estimate of reflector worth. It operates at room temperature using a controlled water loop with secondary and tertiary circuits, two heat exchangers and an electrical heater.

\footnotetext{
Corresponding author: vincent.lamirand@epfl.ch
} 


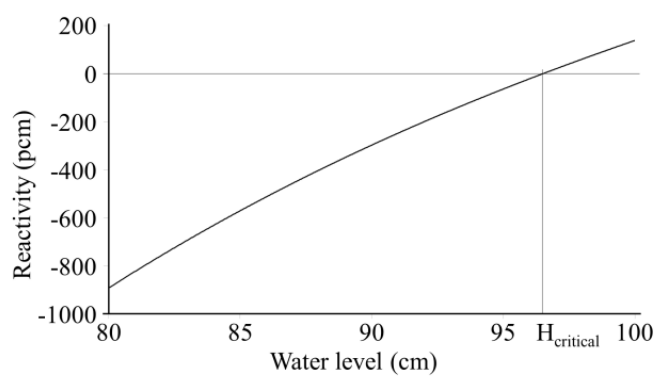

Fig. 1. Reactivity dependence on water level around criticality.

The core is located in an Al-6060 grade vessel of $130 \mathrm{~cm}$ in diameter, $160 \mathrm{~cm}$ in height, and $1.2 \mathrm{~cm}$ in thickness (see Fig. 2). The vessel is filled with demineralized light water which is used as both a moderator and reflector. The core active part has the approximate shape of a cylinder of $100 \mathrm{~cm}$ in height and about $60 \mathrm{~cm}$ in diameter. It consists of two interlocked fuel zones with square lattices of different pitches:

- an inner zone of $336 \mathrm{UO}_{2}$ rods, 1.806 wt.\% enriched, and with a pitch of $1.837 \mathrm{~cm}$,

- an outer zone of $172 \mathrm{U}_{\text {metal }}$ rods in nominal configuration, $0.947 \mathrm{wt} \%$ and $2.917 \mathrm{~cm}$,

- a varying water gap between the two zones because of the two different pitches.

Both uranium fuels consist of a 1-m pile of cylindrical pellets cladded in aluminium. The rods are maintained vertically by two octagonal aluminium grid plates spaced $1 \mathrm{~m}$ apart. In this configuration, the grids have a $0.5-\mathrm{mm}$ cadmium layer to limit axial neutron leakage to the environment, i.e. structure activation, with the active zone of the fuel starting above the lower cadmium layer.

The reactor possesses six independent shutdown mechanisms to bring it to a sub-critical state in less than one second: two cruciform $\mathrm{Cd}$ safety blades in the inner zone; and four expansion tanks to drain the moderator, set in the vessel corners and controlled each by a valve.

\subsection{Metal reflectors}

Following the feasibility study [1], each metal reflector is composed of eight $2-\mathrm{cm}$ thick sheets of $30 \times 30 \mathrm{~cm}^{2}$. These latter ones are made of stainless steel (nucleargrade low cobalt 304L type), natural iron $(99.85 \%$ ), nickel $(99.7 \%)$, and chromium $(99.95 \%)$. Their widths were chosen to limit detection of thermal neutrons scattered in the surrounding water, while keeping a reasonable size for limiting production constraints, cost, and in-core volume. Activation foils shall be positioned between the sheets with great precision, as the flux gradient is large. The metal reflector will be placed in the northwest periphery of the core in a mobile aluminium case, which is currently under design at CEA Cadarache (see Fig. 2). It will allow reproducible positioning as well as easy insertion and extraction of the sheets and dosimeters. Moreover, the case will prevent perturbations from water slipping between the sheets.
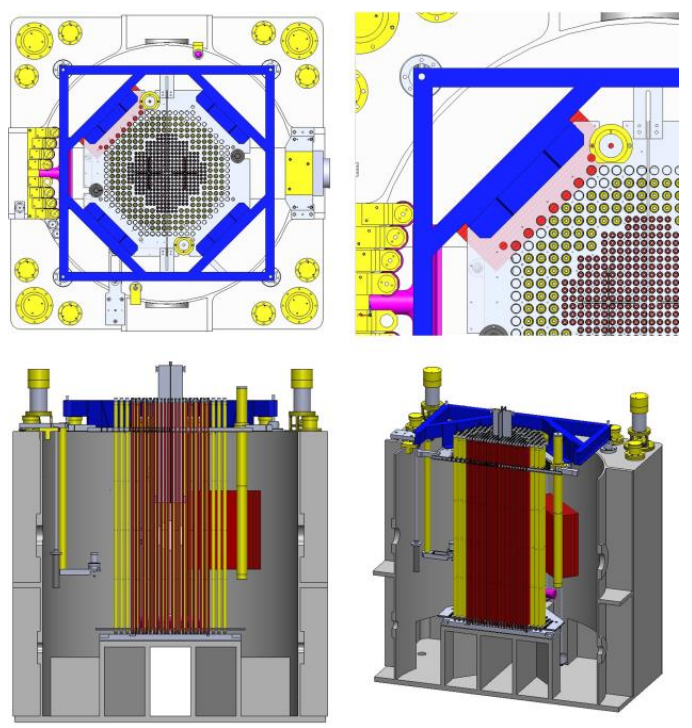

Fig. 2. Model of the metal reflector northwest positioning with respect to the core (top and side views).

\subsection{Gamma spectrometry of activated foils}

After irradiation, the dosimeters' activities will be measured using high-purity germanium (HPGe) gamma spectrometers to be compared with calculation results. The LRS team owns several HPGe detector stations, including a recent Canberra station (detector: GC4518/S; shielding: VG-BB-98/16D1-2), whose efficiency would suffice to measure most activation dosimeters sensitive to the thermal energy range. Regarding dosimeters with low activity, other possibilities are prospected for accurate measurements, e.g. counting in remote lowradioactivity laboratories.

\section{Optimization of dosimetry experiments}

As a zero-power light water reactor, CROCUS is limited in power and in fast neutron flux component. Moreover, its use for education purposes requires the accessibility to its cavity for most of the year to students or, more generally, to visitors. The shutdown configuration has no in-core moderator and, therefore, calls for limited residual dose of the core, i.e. limited integrated power. These two reasons were the main object of the feasibility study [1], while the study presented hereafter focuses on the optimization of the experiments. In addition, although it was proven that dosimeters of interest could indeed be activated, the consequent constraint on nuclear data was not quantified.

The optimization was carried out using Total Monte Carlo uncertainty propagation in association with a Correlated Sampling technique, implemented in a modified version of the Serpent2 Monte Carlo code. The TMC-CS method and the optimization results are presented hereafter. 


\subsection{Total Monte Carlo with Correlated Sampling}

The TMC method consists of sampling distributions of quantities of interest to observe their impact on a system, and consequently propagate their uncertainties. Correlated sampling is a numerical method for Monte Carlo particle transport that allows to maintain the representativeness of two systems using a single particle track. [19] For each sampled event, its probability is calculated for a reference, and for a system modified with regards to the quantity of interest. By the use of weighting for each event, and correction of the final results, score representatives of both systems are obtained in one calculation only. An implementation of CS in the Serpent2 code is available in [20] for thermal feedback estimations. Its coupling to the TMC methods allows reduction of the computational cost.

In the case of the study presented here, the TMC-CS method was used to propagate uncertainties of the nuclear cross sections of interest to the reactions rates in the reflectors dosimeters. By sampling over cross sections distributions representative of their uncertainties, such as random ACE files provided by the TENDL-2017 library, we obtained the associated expected reaction rates distribution in the reflector foils. The development and validation of the method for the present application is presented in [21].

Two examples of reaction rates distributions are presented in Fig. 3, as a function of the dosimeter position in the reflector: position 1 corresponds to the front of the reflector, 2 to the position after the first sheet of iron, etc. For each position, distributions of absolute and relative values are shown (top and bottom). Capture (thermal range) and inelastic (fast range) reactions on indium-115 are compared (left and right sides). For each position, the distribution allows quantifying the target uncertainty of the dosimeter activation to provide feedback on the cross section. It should be significantly lower than the variation induced by the cross section. In addition, specific trends with distance are observable, which should allow analysis by relative comparison of dosimeters at different positions.

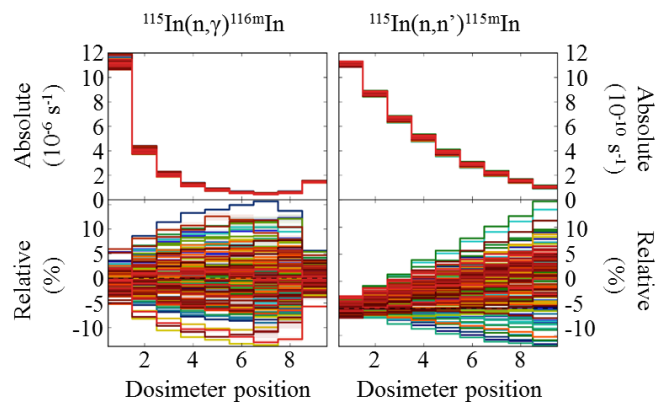

Fig. 3. Distributions of absolute and relative capture (left) and inelastic (right) reaction rates of indium dosimeters with regards to their position in the natural iron reflector. These examples were computed with Serpent 2 using TMC-CS and 128 ACE files from TENDL-2017 representative of the iron cross sections.

\subsection{TMC-CS for design optimization}

Reaction rate distributions were calculated for all three reflector elemental materials and their associated cross sections, and all dosimetry reactions of interest. They were then compared to the expected uncertainties of the dosimeter activities for reasonable irradiation powers and durations ( 1 to $3 \mathrm{~h}$ at $50 \mathrm{~W}$ ), and activity measurements ( 1 to $24 \mathrm{~h}$ ) with the available systems. The calculations are representative of the experimental setup, and take into account self-shielding of dosimeters. Results for all reflector materials are presented in Fig. 4.
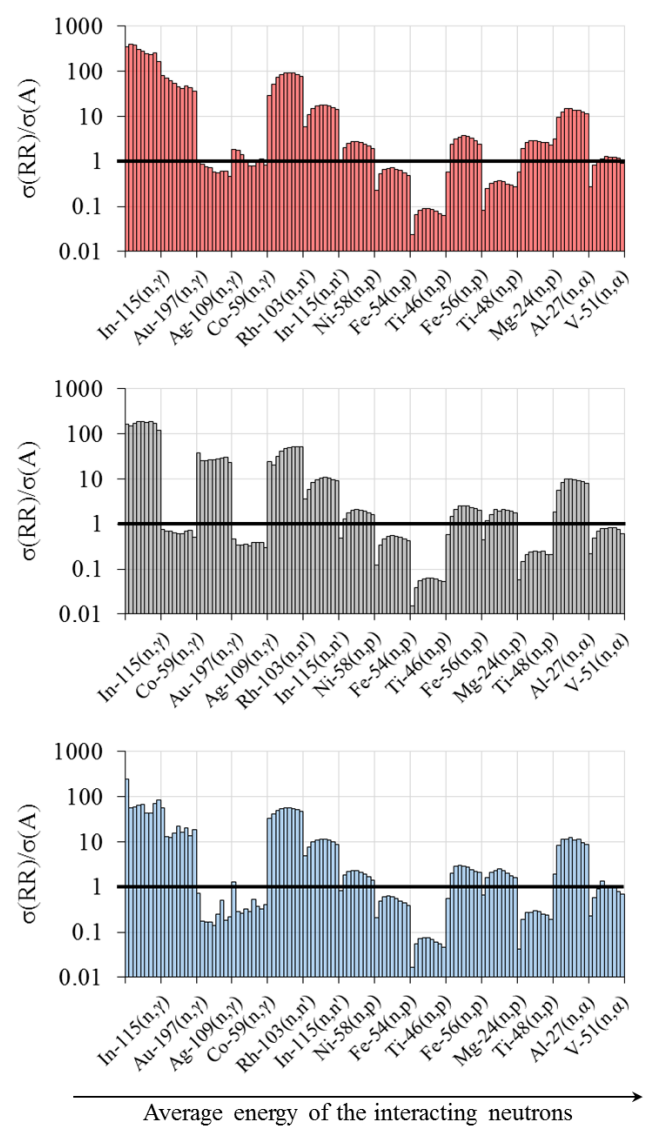

Fig. 4. Ratio of reaction rates' distributions in the elemental reflectors ( $\mathrm{Fe}, \mathrm{Cr}$ and $\mathrm{Ni}$ top to bottom), over uncertainties on activity determination for all considered reactions sorted by lethargy-averaged neutron energy, and for each reaction sorted by dosimeter position.

We observe that, for the range of envisaged irradiations, some reactions are more efficient to set constraints on the nuclear data, consistently over all cases. In a first step, the results provide orders of magnitude that help us to decide which reactions to use for each energy range, and at which position the dosimeters should be set in the reflectors for maximum sensitivity. In the final definition of the experimental programme, the results will help to dimension irradiation specifications for each dosimeter. 
Using TMC-CS, we also quantified the correlations between the reactions' outputs. It allows for us to know which reactions carry redundant or supplementary information about the cross sections. The aim is to choose appropriate redundancy. The results for all reflector types are presented in Fig. 5, reaction by reaction, and for each dosimeter position.
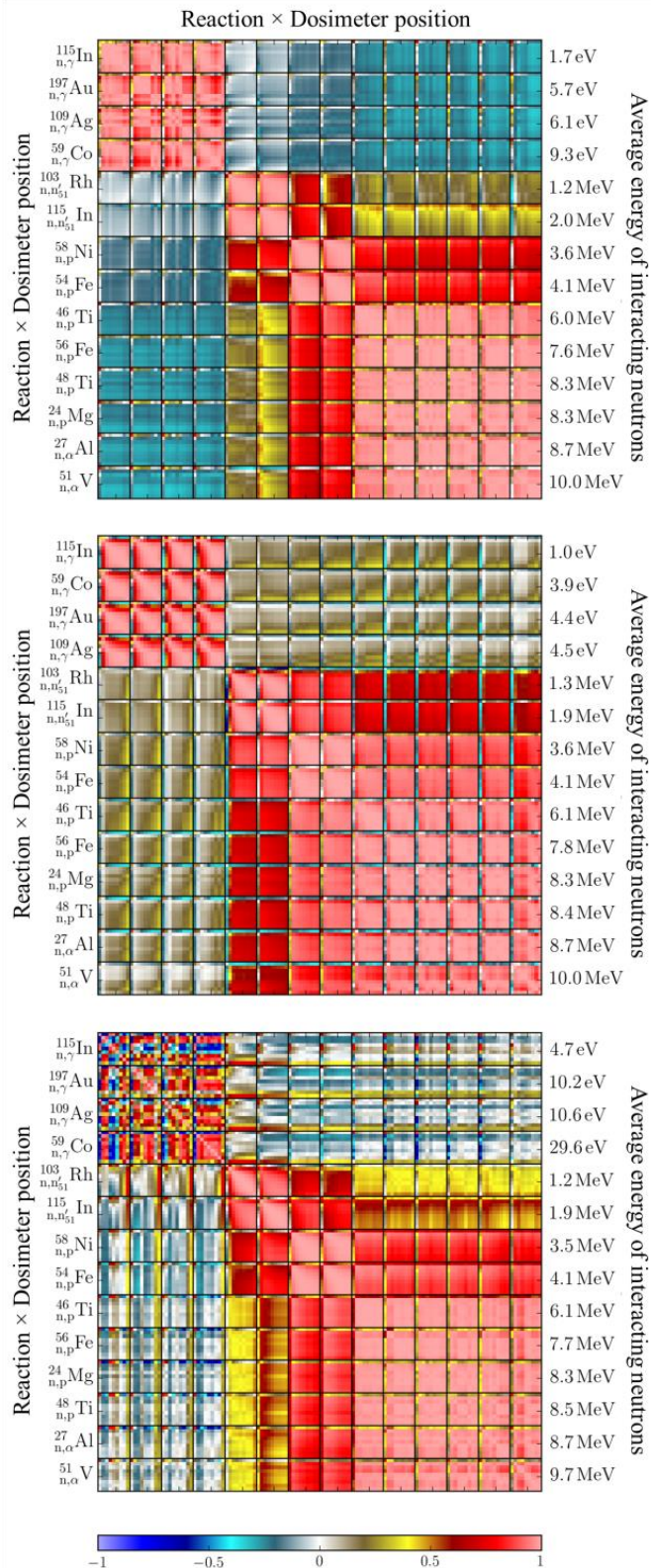

Fig. 5. Correlation matrices of reaction rates for all considered dosimetry reactions in the elemental reflectors $(\mathrm{Fe}, \mathrm{Cr}$ and $\mathrm{Ni}$, top to bottom), sorted by lethargy-averaged neutron energy and dosimeters position ("pixels" within each reaction).
For all three cases, but with variations in intensity, we observe four general groups divided by range of lethargy-averaged energy of the interacting neutrons:

a. eV range: $\operatorname{In}(\mathrm{n}, \gamma), \operatorname{Au}(\mathrm{n}, \gamma), \operatorname{Ag}(\mathrm{n}, \gamma), \operatorname{Co}(\mathrm{n}, \gamma)$,

b. 1-2 MeV range: $\mathrm{Rh}\left(\mathrm{n}, \mathrm{n}^{\prime}\right), \operatorname{In}\left(\mathrm{n}, \mathrm{n}^{\prime}\right)$,

c. $2-5 \mathrm{MeV}$ range: ${ }^{58} \mathrm{Ni}(\mathrm{n}, \mathrm{p}),{ }^{54} \mathrm{Fe}(\mathrm{n}, \mathrm{p})$,

d. above $5 \mathrm{MeV}:{ }^{46} \mathrm{Ti}(\mathrm{n}, \mathrm{p}),{ }^{56} \mathrm{Fe}(\mathrm{n}, \mathrm{p}),{ }^{48} \mathrm{Ti}(\mathrm{n}, \mathrm{p})$, ${ }^{24} \operatorname{Mg}(n, p), \operatorname{Al}(n, \alpha)$ and $\mathrm{V}(\mathrm{n}, \alpha)$.

It should be noted that we obtain a significant sensitivity in the fast range, with no less than three groups carrying information of interest. Still, in the case of the chromium reflector, correlation results present a clear separation between thermal and fast ranges only: all dosimeters in the fast range present maximal correlations, slightly less pronounced for the 1-2 MeV range. Regarding the nickel reflector, correlation oscillations in the thermal range are representative of the low dispersion in the reactions rates induced by the low nuclear data uncertainties. In the prospect of data assimilation, these correlations between dosimeters should be taken into account with great care.

\subsection{Selection of dosimetry reactions}

From the reactions' properties, and with the additional information deduced from the ratios and correlations presented above, we are able to define which reactions should be favoured. For consistency purposes, and as the study confirmed the possibility, we would favour the same set of reactions for all transmission experiments. The list of selected reactions and their ratio values for the iron reflector are presented in Table 1 .

Table 1. Reactions of interest for all transmission experiments, with propagation results in the case of the natural iron reflector. The reactions are sorted by neutron energy range and group of interest, as defined in Section 3.2. The maximum ratios of reaction rate distribution over activity uncertainty are indicated, as well as the associated dosimeter positions in the reflector.

\begin{tabular}{|c|c|c|c|c|}
\hline Group & Reaction & Half-life & $\begin{array}{c}\text { Max. } \\
\text { ratio }\end{array}$ & $\begin{array}{c}\text { Max. } \\
\text { position }\end{array}$ \\
\hline a & ${ }^{115} \operatorname{In}(\mathrm{n}, \gamma)$ & $54.3 \mathrm{~min}$ & 400 & 2 \\
\hline $\mathrm{a}$ & ${ }^{197} \mathrm{Au}(\mathrm{n}, \gamma)$ & $2.70 \mathrm{~d}$ & 79 & 1 \\
\hline $\mathrm{b}$ & ${ }^{115} \mathrm{In}(\mathrm{n}, \mathrm{n})$ & $4.49 \mathrm{~h}$ & 18 & 5 \\
\hline $\mathrm{c}$ & ${ }^{58} \mathrm{Ni}(\mathrm{n}, \mathrm{p})$ & $70.9 \mathrm{~d}$ & 2.7 & 4 \\
\hline $\mathrm{c}$ & ${ }^{54} \mathrm{Fe}(\mathrm{n}, \mathrm{p})$ & $312 \mathrm{~d}$ & 0.7 & 5 \\
\hline $\mathrm{d}$ & ${ }^{56} \mathrm{Fe}(\mathrm{n}, \mathrm{p})$ & $2.58 \mathrm{~h}$ & 3.7 & 5 \\
\hline $\mathrm{d}$ & ${ }^{27} \mathrm{Al}(\mathrm{n}, \alpha)$ & $15.0 \mathrm{~h}$ & 15 & 4 \\
\hline
\end{tabular}


We observe high ratios for several reactions, both in the thermal and fast ranges, indicating space for minimisation of the irradiation power and duration. Reactions with low ratios but long half-lives, such as ${ }^{58} \mathrm{Ni}(\mathrm{n}, \mathrm{p})$ and ${ }^{54} \mathrm{Fe}(\mathrm{n}, \mathrm{p})$, are to be favoured for measurements in remote laboratories. In addition, we observe that some dosimetry materials used in their natural abundance, i.e. In and $\mathrm{Fe}$, should provide complementary information of interest for our set of experiments by using different reactions, and without increasing the number of irradiations. As the position of maximum sensitivity varies with the reaction, irradiations with mixed dosimeter types are also to be considered. In the specific case of the ${ }^{103} \mathrm{Rh}(\mathrm{n}, \mathrm{n}$ ') reaction, and despite its high ratio, it was discarded because of the difficulty to measure the low energy X-ray emission coupled with a short half-life (56 min), preventing measurements in remote laboratories. Another result of this study is the confirmation of the interest of nickel and iron dosimeters: it validates the principle of mapping the activity after irradiation of the corresponding reflector sheets themselves.

\section{Conclusion}

In the present article, we described an optimization study carried out for the design of PETALE's transmission experiments on stainless steel and its main elements. It used a new uncertainty propagation method based on Total Monte Carlo coupled with Correlated Sampling using Serpent2 and TENDL-2017. Because of the flux and operational constraints of the CROCUS facility, quantification and optimization of the impact of the experiments on target nuclear data was required. It concluded on an optimized set of dosimetry reactions namely ${ }^{115} \operatorname{In}(\mathrm{n}, \gamma), \quad{ }^{197} \mathrm{Au}(\mathrm{n}, \gamma), \quad{ }^{115} \operatorname{In}(\mathrm{n}, \mathrm{n}), \quad{ }^{58} \mathrm{Ni}(\mathrm{n}, \mathrm{p})$, ${ }^{54} \mathrm{Fe}(\mathrm{n}, \mathrm{p}),{ }^{56} \mathrm{Fe}(\mathrm{n}, \mathrm{p}),{ }^{27} \mathrm{Al}(\mathrm{n}, \alpha)-$, and target uncertainties for defining irradiation and measurements conditions. The next step is to propagate the uncertainties from technological parameters and the cross sections of the dosimetry reactions, in order to finalise the experimental setup and to define the experimental programme.

The authors thank Mathieu Hursin and Daniel Siefman for their assistance and feedback on uncertainty quantification and propagation.

\section{References}

1. V. Lamirand, G. Perret, S. Radman, D. Siefman, M. Hursin, P. Frajtag, A. Gruel, P. Leconte, P. Blaise, and A. Pautz, React. Dosim. 16th Int. Symp. ASTM STP1608, 7 (2018).

2. D. Siefman, M. Hursin, D. Rochman, S. Pelloni, and A. Pautz, Eur. Phys. J. Plus 133, 429 (2018)

3. A. J. Koning and D. Rochman, Ann. Nucl. Energy 35, no. 11, 2024-2030 (2008).

4. A. J. Koning and D. Rochman, Nucl. Data Sheets, 113, no. 12, 2927-2934 (2012)

5. J. Leppänen et al., Ann. Nucl. Energy 82, 142-150 (2015)
6. A. Rais, D. Siefman, G. Girardin, M. Hursin, and A. Pautz, IGORR 2014 (2014)

7. M. Hursin, A. Rais, D. Siefman, G. Girardin, and A. Pautz, International Technical Meeting on Small Reactors (2014)

8. O. Pakari, V. Lamirand, G. Perret, D. Godat, M. Hursin, P. Frajtag, and A. Pautz, PHYSOR 2018 (2018)

9. V. Lamirand, G. de Izarra, A. Krasa, G. Perret, O. V. Pakari, M. Hursin, P. Blaise, J. Wagemans, and A. Pautz, PHYSOR 2018 (2018)

10. A. Rais, D. Siefman, G. Girardin, M. Hursin, and A. Pautz, Sci. Technol. Nucl. Install. (2015)

11. D. J. Siefman, G. Girardin, A. Rais, A. Pautz, and M. Hursin, Ann. Nucl. Energy 85, 434-443 (2015)

12. A. Rais, M. Hursin, G. Perret, and A. Pautz, PHYSOR 2016 (2016)

13. V. Lamirand, M. Hursin, G. Perret, P. Frajtag, O. Pakari, and A. Pautz, RRFM/IGORR 2016, 284-292 (2016)

14. A. Rais, Ecole Polytechnique Fédérale de Lausanne (EPFL) (2017)

15. V. Lamirand, A. Gruel, G. Perret, P. Leconte, P. Frajtag, M. Hursin, P. Blaise, and A. Pautz, ISRD16 (2017)

16. O. Pakari, V. Lamirand, G. Perret, and A. Pautz, ANIMMA 2017 (2017)

17. O. Pakari, V. Lamirand, G. Perret, P. Frajtag, and A. Pautz, IEEE Trans. Nucl. Sci. 65, no. 9, 2456-2460 (2018).

18. U. Kasemeyer, R. Früh, J. M. Paratte, and R. Chawla, International Reactor Physics Experiments Handbook (IRPhE) 4440, OECD, Ed. 2007, 94 (2007)

19. H. Rief, Ann. Nucl. Energy 11, no. 9, 455-476 (1984)

20. A. Laureau, L. Buiron, and B. Fontaine, EPJ Nucl. Sci. Technol. 3, 17 (2017)

21. A. Laureau, V. Lamirand, D. Rochman, and A. Pautz, WONDER 2018 (2018) 